\title{
Treatment of initial parenchymal central nervous system involvement in systemic aggressive B-cell lymphoma
}

\author{
Marcel Nijland, Anne Jansen, Jeanette K. Doorduijn, Roelien H. Enting, \\ Jacoline E. C. Bromberg \& Hanneke C. Kluin-Nelemans
}

To cite this article: Marcel Nijland, Anne Jansen, Jeanette K. Doorduijn, Roelien H. Enting, Jacoline E. C. Bromberg \& Hanneke C. Kluin-Nelemans (2017): Treatment of initial parenchymal central nervous system involvement in systemic aggressive B-cell lymphoma, Leukemia \& Lymphoma, DOI: 10.1080/10428194.2017.1285026

To link to this article: http://dx.doi.org/10.1080/10428194.2017.1285026

+ View supplementary material $\longleftarrow$

Published online: 06 Feb 2017.

Submit your article to this journal $₫$

山 Article views: 9

Q View related articles $\longleftarrow$

View Crossmark data $\llbracket$ 


\title{
Treatment of initial parenchymal central nervous system involvement in systemic aggressive B-cell lymphoma
}

\author{
Marcel Nijland ${ }^{a}$, Anne Jansen ${ }^{b}$, Jeanette K. Doorduijn ${ }^{c}$, Roelien H. Enting ${ }^{d}$, Jacoline E. C. Bromberg ${ }^{e}$ and \\ Hanneke C. Kluin-Nelemans ${ }^{a}$ \\ ${ }^{\mathrm{a} D e p a r t m e n t}$ of Haematology, University Medical Centre Groningen, University of Groningen, Groningen, Netherlands; ${ }^{\mathrm{b}}$ Department of \\ Neurology, Canisius-Wilhelmina Hospital, Nijmegen, Netherlands; 'Department of Haematology, Erasmus MC Cancer Institute, \\ Rotterdam, Netherlands; ${ }^{d}$ Department of Neurology, University Medical Centre Groningen, University of Groningen, Groningen, \\ Netherlands; ${ }^{e}$ Department of Neurology, Erasmus MC, Rotterdam, Netherlands
}

\section{ABSTRACT}

Central nervous system (CNS) involvement in systemic B-cell non-Hodgkin lymphoma (B-NHL) at diagnosis (sysCNS) is rare. We investigated the outcome of 21 patients with sysCNS, most commonly diffuse large B-cell lymphoma, treated with high dose methotrexate (HD-MTX) and R-CHOP. The median number of cycles of HD-MTX and R-CHOP was 4 (range 1-8) and 6 (range $0-8)$, respectively. Consolidative whole brain radiotherapy (WBRT) was given to $33 \%(7 / 21)$ patients. With a median follow-up of 44 months the 3-year progression free survival (PFS) and overall survival (OS) were $45 \%(95 \% \mathrm{Cl} 34-56 \%)$ and $49 \%(95 \% \mathrm{Cl} 38-60 \%)$, respectively. Over $90 \%$ of patients had an unfavorable international prognostic index score, reflected by treatmentrelated mortality of $19 \%(4 / 21)$ and relapse-related mortality of $28 \%(6 / 21)$. The outcome of these patients was, however, unexpectedly good when compared to secondary CNS relapses. Prospective studies are needed to define the optimal treatment for patients with sysCNS, but its rarity might be challenging.

\section{ARTICLE HISTORY}

Received 24 October 2016 Revised 10 January 2017

Accepted 15 January 2017

\section{KEYWORDS}

Aggressive B-cell lymphoma; systemic; central nervous system; methotrexate; R-CHOP

\section{Introduction}

The risk of central nervous system (CNS) dissemination of aggressive B-cell lymphoma (B-NHL) is 5\% [1]. High international prognostic index (IPI) score, kidney or adrenal gland involvement and diffuse large B-cell lymphoma (DLBCL) with dual expression of MYC and BCL2 by immunohistochemistry convey a high risk of CNS relapse [2,3]. In the majority, CNS involvement occurs during progression of the disease. Rarely, CNS dissemination is already present at initial diagnosis. Unlike the general favorable outcome of patients with primary CNS lymphoma (PCNSL) when treated with high dose methotrexate (HD-MTX), cytarabine and thiothepa [4-7], CNS relapse of aggressive B-NHL conveys a dismal prognosis [8,9]. Salvage regimens with relapsed PCNSL incorporating thiothepa and high dose chemotherapy followed by autologous stem cell transplantation (ASCT) showed an improved overall survival (OS) for these patients if initially responsive and subsequently eligible for intensive treatment $[10,11]$.
It is unknown if the prognosis of concomitant systemic and parenchymal CNS B-NHL at diagnosis (sysCNS B-NHL) is different from secondary CNS lymphoma in the relapse setting. Given its rarity, data on treatment for sysCNS B-NHL are sparse and there is no established standard of care. Recent data from two studies suggest a beneficial effect of intensive chemotherapy followed by ASCT $[12,13]$. In both studies, ASCT was only offered to patients in complete remission (CR) (31\%-50\%). Furthermore, in one of the studies, anthracyclines - highly effective in systemic B-NHL - were only a minor part of the induction regimen [13]. Outcome of non-transplant strategies in sysCNS B-NHL was only explored in small series $[14,15]$.

We observed an excellent response in a patient with sysCNS initially treated as if primary CNS with the European Organisation for Research and Treatment of Cancer (EORTC) regimen MBVP (methotrexate, etoposide, carmustine and prednisolone) [5]. In this patient, not only the CNS component responded well, but also

CONTACT Marcel Nijland m.nijland@umcg.nl E Internist-Haematologist, Department of Haematology, University Medical Centre Groningen, Hanzeplein 1, 9713 GZ Groningen, Netherlands 
systemic involvement responded at the same time. Consequently, we continued after MBVP with rituximab, cyclophosphamide, doxorubicin, vincristine and prednisolone (R-CHOP), and applied this regimen to the next series of patients. Simultaneously, another University Medical Center in the Netherlands combined R-CHOP alternating with HD-MTX, based upon the same observations.

Therefore, we retrospectively investigated the outcome of patients with sysCNS B-NHL treated in both centers with a combination of drugs effective for CNS and systemic involvement. None of these patients underwent ASCT.

\section{Methods}

\section{Study design and patient identification}

Clinical data on patients with sysCNS B-NHL were retrieved from the electronical databases of two referral medical centres in the Netherlands. All patients had newly diagnosed, histologically proven aggressive B-cell lymphoma. In case of transformed lymphoma, patients had not received prior therapy. CNS involvement was determined either by biopsy, magnetic resonance imaging (MRI) and/or CSF examination. Patients had been treated between 2000 and 2015. Patients should have received at least one cycle of HD-MTX. Both regimens start with a cycle of HD-MTX. The pathological assessment was performed by experienced hematopathologists. Since patients were treated according to best practice no medical ethical committee approval was required.

\section{Treatment and follow-up}

\section{Initial assessment}

Staging consisted of computed tomography scan of neck, chest, abdomen and pelvis, bone marrow biopsy, cerebrospinal fluid (CSF) examination with four-color flow cytometry (FCM), MRI brain, and further investigations if clinically indicated. A fluorodeoxyglucose $\left({ }^{18} \mathrm{~F}-\mathrm{FDG}\right)$ positron emission tomography scan was available for all patients treated at one of the medical centres. Systemic B-NHL was diagnosed by lymph node or tissue biopsies in all cases. CNS involvement was diagnosed either by brain biopsy or CSF flow cytometry in 58\% (12/21) of patients. In 42\% (9/21) neurological symptoms and parenchymal abnormalities on the MRI scan were considered sufficient for the diagnosis of CNS involvement. In 10\% (2/21) CNS involvement presented as neurolymphomatosis. In all patients the international prognostic index (IPI) was documented.

\section{Treatment}

Patients were treated according to one of two regimens. At the University Medical Centre Groningen a sequential regimen was adopted. Four cycles of MTX at a dose of $3 \mathrm{~g} / \mathrm{m}^{2}$ were given as part of the MBVP regimen, which also includes carmustine $\left(100 \mathrm{mg} / \mathrm{m}^{2}\right.$ at day 4$)$, teniposide $\left(100 \mathrm{mg} / \mathrm{m}^{2}\right.$ at day 2 and 3$)$ and prednisolone $\left(60 \mathrm{mg} / \mathrm{m}^{2}\right.$ at day 1-5) [5]. Interim response evaluation was performed after completion of HD-MTX. Patients with a partial CNS remission received whole brain radiotherapy (WBRT) at this point. Patients subsequently continued for 6 cycles of R-CHOP after which end-of-treatment evaluation took place. R-CHOP was given as a combination of rituximab ( $375 \mathrm{mg} / \mathrm{m}^{2}$ at day 1$)$, cyclophosphamide $\left(750 \mathrm{mg} / \mathrm{m}^{2}\right.$ at day 1$)$, doxorubicin $\left(50 \mathrm{mg} / \mathrm{m}^{2}\right.$ at day $1)$, vincristine $\left(1.4 \mathrm{mg} / \mathrm{m}^{2}\right.$ at day 1 up to a maximal dose of $2 \mathrm{mg}$ ) and prednisolone (100 $\mathrm{mg}$ at day 1-5). At the Erasmus Medical Centre Rotterdam an alternating regimen was adopted, which consisted of 6 alternating cycles of HD-MTX at a dose of $3 \mathrm{~g} / \mathrm{m}^{2}$ and R-CHOP. Interim evaluation was performed after 3 cycles and end-of-treatment evaluation after completion of therapy. Patients with a partial remission (PR) of the CNS involvement received WBRT. In both regimens patients with leptomeningeal involvement received intrathecally methotrexate and/or cytarabine until complete clearance of the CSF as assessed by FCM. Furthermore, 4 patients in the alternating regimen received i.t. therapy without a positive CSF because of local practice.

\section{Response assessment}

Response was evaluated according to the 1999 consensus criteria for systemic NHL and the consensus criteria for PCNSL $[16,17]$. Tumor response was classified as $C R$, PR, stable disease (SD), or progressive disease (PD). Only patients in complete remission at CNS and systemic sites at the end of treatment were reported as a CR. Treatment related mortality (TRM) was defined as death during or $\leq 3$ months after treatment.

Follow-up was completed until February 2016.

\section{Statistical analysis}

Duration of follow-up was calculated for all patients alive.The primary endpoints were OS and progression free survival (PFS). OS was defined as time from diagnosis until death (from any cause); PFS as the time 
from diagnosis until relapse, progression or death (from any cause). Survival curves were estimated according to the Kaplan-Meier method. Betweengroup differences in OS and TTP were evaluated using the log-rank test. All categorical variables were expressed as counts and percentages. Where applicable, differences between groups were evaluated by chi-square for binary variables and independent T-tests for continuous variables. A two-tailed $p$-value of less than 0.05 indicated statistical significance. All analyses were performed using IBM SPSS Statistics version 22 (IBM Corp., Armonk, NY).

\section{Results}

\section{Clinical characteristics}

Between 2000 and 201521 sysCNS B-NHL patients were treated. Demographic data from the patients in the two treatment regimens are presented in Table 1. Diffuse large B-cell lymphoma composed the major histological B-NHL entity (66\%). The median age was 54 years (range 19-71 years) with a slight female preponderance. Two-third of patients had a WHO performance score $\geq 2$ and $91 \%$ of patients had an intermediate or high IPI-score. A large number of patients had lymphoma infiltration of organs known to convey a high risk of CNS relapse, like testicles ( $44 \%$ of patients at risk), bone $(38 \%)$, kidney $(19 \%)$ and breast $(17 \%$ of patients

Table 1. Clinical characteristics at diagnosis of the 21 patients with concomitant systemic and parenchymal central nervous system B-cell non-Hodgkin.

\begin{tabular}{|c|c|c|}
\hline Characteristics & $\begin{array}{c}\text { Sequential } \\
\text { MBVP/R-CHOP }\end{array}$ & $\begin{array}{c}\text { Alternating } \\
\text { MTX/R-CHOP } \\
\end{array}$ \\
\hline Patients $(n)$ & 10 & 11 \\
\hline Median age & $54(41-60)$ & $59(19-71)$ \\
\hline Gender $(M / F)$ & $2 / 8$ & $7 / 4$ \\
\hline \multicolumn{3}{|l|}{ B-cell lymphoma } \\
\hline Diffuse large B-cell lymphoma (\%) & $5(50)$ & $9(82)$ \\
\hline latrogenic immunodeficiency lymphoma (\%) & $2(20)$ & $1(9)$ \\
\hline Transformed marginal zone lymphoma (\%) & $2(20)$ & $0(0)$ \\
\hline Intravascular B-cell lymphoma (\%) & $1(10)$ & $1(9)$ \\
\hline \multicolumn{3}{|l|}{ Extra CNS involvement } \\
\hline Testicular (\%) ${ }^{\mathrm{a}}$ & $0(0)$ & $4 / 7(57)$ \\
\hline Breast $(\%)^{a}$ & $2 / 8(25)$ & $0(0)$ \\
\hline Bone/bone marrow (\%) & $6(60)$ & $2(18)$ \\
\hline Kidney/lung (\%) & $4(40)$ & $0(0)$ \\
\hline Cerebrospinal fluid involvement (\%) & $4(40)$ & $4(45)$ \\
\hline Elevated LDH (\%) & $6(60)$ & $6(54)$ \\
\hline \multicolumn{3}{|l|}{ WHO performance } \\
\hline $1-2(\%)$ & $2(20)$ & $5(45)$ \\
\hline $3-4(\%)$ & $8(80)$ & $6(55)$ \\
\hline \multicolumn{3}{|l|}{ IPI-score } \\
\hline $1(\%)$ & $1(10)$ & $1(9)$ \\
\hline $2-3(\%)$ & $7(70)$ & 7 (64) \\
\hline 4-5 (\%) & $2(20)$ & $3(27)$ \\
\hline
\end{tabular}

${ }^{\text {a }}$ Percentage in patients at risk.

Testicular involvement can only be observed in male patients. Breast involvement can only be observed in female patients. at risk). Besides parenchymal CNS, concomitant CSF involvement was present in $43 \%$ of patients.

\section{Treatment}

Thirteen patients (62\%) completed therapy with only minor deviations. Two patients received less than $6 \mathrm{R}$ $\mathrm{CHOP}$ cycles. One patient had both a reduction in the number of MTX and R-CHOP cycles. Data on treatment intensity between the two treatment regimens are presented in Table 2. Detailed information on individual treatment and response assessment is shown in Supplementary Figure 1. The median number of HDMTX cycles was 4 (range 1-8). Thirteen patients (62\%) received concomitant intrathecal MTX. The median number of R-CHOP cycles was 6 (range 0-8). WBRT was given to 7 patients (33\%), all because of persistent abnormalities on brain MRI. One patient received local radiotherapy on an extracranial site because of PR at the end of treatment. Besides the additional VP16 and $B C N U$ in patients treated with MBVP, there was no significant difference between the two regimens.

\section{Follow-up, response and survival}

The median duration of follow-up of patients was 44 months (range 5-104). The overall response rate (ORR) at the end of treatment was $62 \%:$ CR $57 \%(n=12)$ and PR $5 \%(n=1)$. PD was noted in $19 \%(n=4)$, whereas early TRM due to infectious complications was observed in $19 \%(n=4)$. All infectious complications occurred during neutropenia. Two patients died as a consequence of an aspiration pneumonia due to neurogenic dysphagia. One patient developed a complicated clostridium difficile infection after prolonged treatment with ciprofloxacin. One patient died of a complicated meningitis (Klebsiella pneumoniae), which was not related to a previous lumbar puncture. One patient died 6 months after treatment as a result of cognitive deterioration.

Table 2. Treatment intensity in the 21 patients with concomitant systemic and CNS B-NHL at diagnosis.

\begin{tabular}{lcc}
\hline Characteristics & $\begin{array}{c}\text { Sequential } \\
\text { MTX/R-CHOP }\end{array}$ & $\begin{array}{c}\text { Alternating } \\
\text { MTX/R-CHOP }\end{array}$ \\
\hline Patients $(n)$ & 10 & 11 \\
Median MTX cycles $(n)$ & $4(2-6)$ & $5(1-8)$ \\
Median cumulative MTX dosing (gr) & $12(6-18)$ & $15(3-24)$ \\
Median i.t. MTX cycles $(n)^{\mathrm{a}}$ & $6(4-10)$ & $9(4-13)$ \\
Median CHOP cycles $(n)$ & $6(0-6)$ & $6(1-8)$ \\
Rituximab (\%) & $10(100)$ & $10(90)$ \\
Additional chemotherapy & Teniposide Carmustine & n.a. \\
Radiotherapy in PR $(\%)$ & $3(30)$ & $4(36)$ \\
\hline
\end{tabular}

an patients receiving i.t. therapy.

Number of i.t. cycles only described for patients receiving intrathecal therapy. 

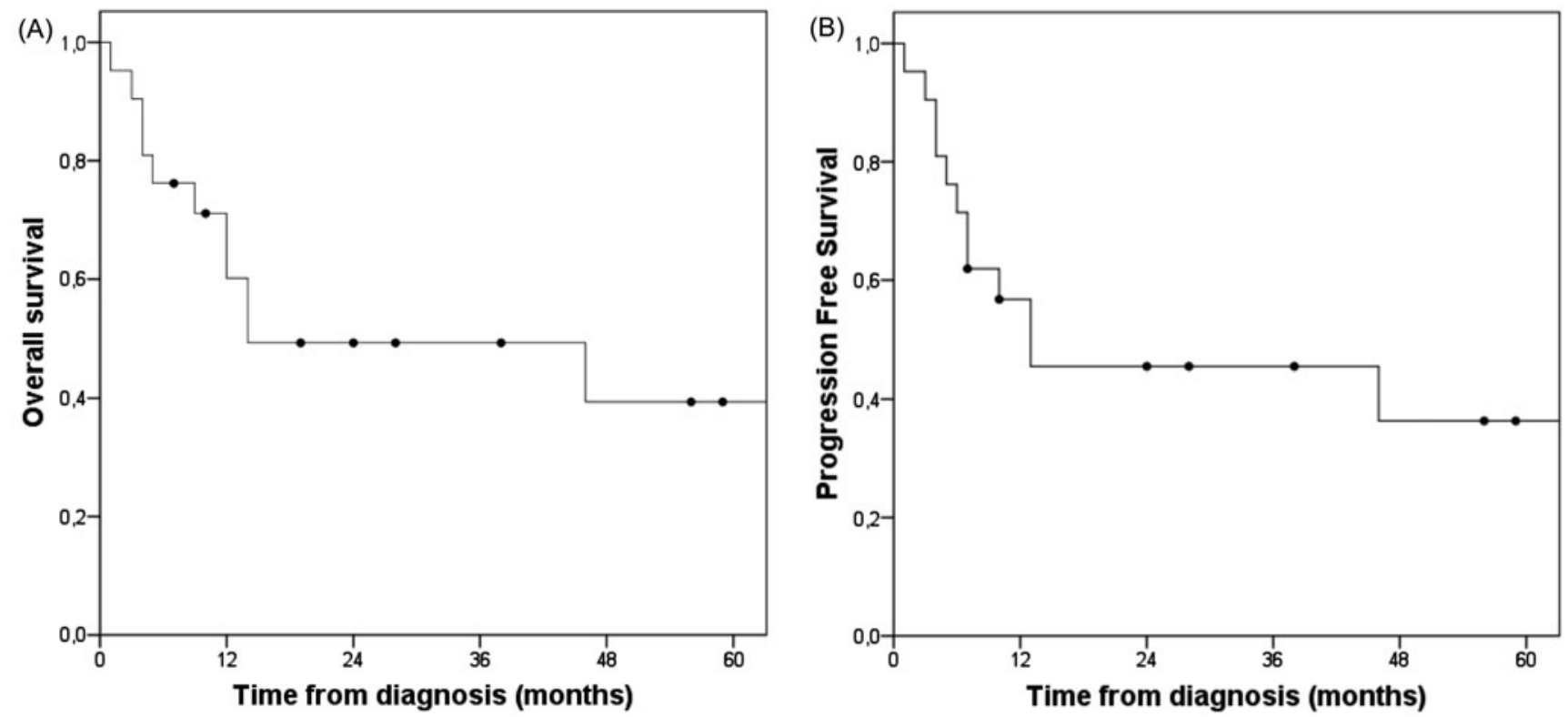

Figure 1. (A) OS and (B) PFS of 21 patients with concomitant systemic and parenchymal CNS B-NHL at diagnosis.

Three-year PFS and OS for the entire cohort were $45 \%(95 \% \mathrm{Cl} 34-56 \%)$ and $49 \%(95 \% \mathrm{Cl} \quad 38-60 \%)$, respectively (Figure $1(A, B)$ ). No significant differences in PFS and OS were found between both regimens (HR OS 1.25, 95\% Cl 0.38-4.2; $p$ 0.72). Although statistical analysis showed no significant differences in outcome between the various variables due to the small number of patients, all patients $(n=3)$ with IPI-score 0-1 were alive at follow-up, whereas all patients $(n=4)$ with IPI-score $4-5$ died within 2 years after diagnosis.

The combined incidence of refractory and relapsed sysCNS was $28 \%$ (6 of 21 patients). The relapse rate in patients achieving a CR was only $8 \%$ ( 1 of 12 patients), which is reflected by the plateau after 12 months in the PFS and OS curves (Figure 1(A,B)). Patients in CR/ PR had a 3-year PFS and OS of $74 \%$ and $83 \%$, respectively (Supplementary Figure $2(A, B)$ ) The patient who relapsed did so within a year in the CNS. She subsequently received WBRT but died soon afterwards. The one patient in PR progressed after 8 months with both systemic and CNS involvement. He received no further therapy and died shortly thereafter. The four patients with PD all died within 6 months. The site of progression was systemic-only in two patients, CNS-only in one patient and concomitant systemic and CNS in one patient.

\section{Discussion}

By combining the results from two medical referral centres in the Netherlands, we performed the second largest retrospective study in patients with sysCNS
B-NHL. Findings from this study indicate that nontransplant regimens of HD-MTX and R-CHOP can be an effective treatment for sysCNS B-NHL. With a 3-year OS of $49 \%$ the outcome in our cohort is comparable to the more intensive regimen including ASCT, which is available to only a selected group of patients (5-year OS 41\%) [13]. In one of the three studies with nontransplant regimens 4 out of $6(67 \%)$ patients achieved a CR when treated with HD-MTX and R-CHOP. In a second study with HD-MTX, rituximab, cytarabine and idarubicin (R-IDARAM) 4 out of 10 (40\%) patients achieved a CR. Median OS of both studies was 25.1 months and more than 30 months, respectively $[14,18]$. In a pilot study with dose-adjusted EPOCH (etoposide, cyclophosphamide, doxorubicin, vincristine, prednisone) plus rituximab 8 out of 8 patients achieved a CR [15]. Four patients underwent a consolidative autologous stem cell transplantation. With a median follow up of 11 months all patients were alive.

In most of our patients systemic involvement included one or more organs known to convey a high risk of CNS involvement in systemic B-NHL [1]. In particular 4 patients had renal involvement, which is the strongest risk factor for CNS relapse in DLBCL [3]. These findings suggest an early dissemination of a systemic B-NHL, rather than a systemic manifestation of a PCNSL. This might be important, since PCSNL has several pathological features that are only infrequently present in systemic DLBCL, such as a high incidence of mutations in $C D 79 B$ and MYD88, loss of human leukocyte adhesion (HLA) molecules and frequent expression of the programed death ligand 1 [19-21]. Despite its similarity with systemic B-NHL, the OS of sysCNS 
B-NHL treated with HD-MTX and R-CHOP was better than expected and comparable to that of PCNSL and certainly not as bad as secondary relapsed CNS B-NHL treated with HD-MTX and cytarabine (Supplementary Table 1) $[4-6,8]$.

The TRM in our cohort of $19 \%$ was high, and reflects the very unfavorable risk profile of our patients with $67 \%$ having a WHO performance of $3-4$ and $14 \%$ being older than 65 -years. Given the small numbers it cannot be answered whether the concomitant systemic involvement or additional R-CHOP played a role.

Despite the limited number of relapses, two observations were interesting: firstly, all but one patient who relapsed did so less than 6 months after the end of treatment. All other patients who failed did so during the early phase of treatment; secondly, in refractory and relapsing patients the majority had systemic progression. The incidence of refractory/early progressing patients in our cohort (24\%) was similar to that of more intensive regimens (35\%) [13]. This means that progressive patients have a dismal outcome and are unlikely to be salvaged. It is interesting to speculate whether drugs such as bendamustine, ibrutinib, checkpoint inhibitors and lenalidomide would be effective in this setting, since these drugs generally have a favorable toxicity profile, known activity in relapsed systemic B-NHL and CNS penetration [22-26].

Notably, patients who obtain a CR have an excellent outcome and probably will not benefit from additional high dose therapy, especially those patients with a low IPI at start. This challenges the role of consolidation as suggested by the French lymphoma group LYSA, that showed a positive impact of high-dose therapy followed by autologous stem cell transplantation on 3year OS and PFS [12]. However, in our series 7 of 21 patients (33\%) received consolidative radiotherapy. The indirect answer if ASCT can replace WBRT will have to come from the analysis of the IELSG32 trial, where patients with a chemosensitive PCNSL were randomized in a second randomization to WBRT or ASCT [7]. But even then we have to be cautious to extrapolate these findings to sysCNS, since PCSNL has a different biology.

Our study obviously has limitations. Firstly, its retrospective nature has inherent known and unknown biases. We have no information on the outcome of those patients with synCNS lymphoma who were considered too frail for referral to our university centers. This means that our data might be based upon a selection of those patients fit for high dosed methotrexate. Furthermore, although the rationale behind the treatment was the same, e.g. the combination of primary CNS treatment followed by systemic immunochemotherapy, there was a considerable heterogeneity in the treatment received. Some patients were treated with a sequential MBVP/R-CHOP and others with an alternating HD-MTX/R-CHOP regimen. Although we observed no significant difference between the regimens in OS, we cannot assess the separate effect of teniposide and carmustine. In addition, 7 of 21 patients were unable to complete treatment as planned (Supplementary Figure 1). Except for gender and associated testicular or breast localization we observed no significant differences in clinical characteristics or outcome. Given its rarity, sysCNS B-NHL comparative studies can only be performed in a large, international multicentre setting. Lastly, high dose cytarabine and thiothepa were not part of the treatment regimens, although in PCNSL the addition improved outcome in a randomized phase II study [7]. Whether the addition of cytarabine or thiothepa in sysCNS B-NHL is feasible remains to be established, but might be offset by the TRM.

\section{Conclusions}

In this second largest cohort of patients with sysCNS lymphoma at presentation described to date, the outcome of patients treated with HD-MTX and R-CHOP was excellent for those patients who obtained a complete remission. This challenges the fact that these patients are excluded from most trials, and usually are considered as having a dismal prognosis. Prospective studies are needed to define the optimal treatment for these patients.

Potential conflict of interest: Disclosure forms provided by the authors are available with the full text of this article online at http://10.1080/10428194.2017. 1285026.

\section{References}

[1] Ferreri AJ, Assanelli A, Crocchiolo R, et al. Central nervous system dissemination in immunocompetent patients with aggressive lymphomas: incidence, risk factors and therapeutic options. Hematol Oncol. 2009;27:61-70.

[2] Savage KJ, Slack GW, Mottok A, et al. Impact of dual expression of MYC and BCL2 by immunohistochemistry on the risk of CNS relapse in DLBCL. Blood. 2016;127:2182-2188.

[3] Schmitz N, Zeynalova S, Nickelsen M, et al. CNS international prognostic index: a risk model for CNS relapse in patients with diffuse large B-cell lymphoma treated with R-CHOP. J Clin Oncol. 2016;34:3150-3156.

[4] Gavrilovic IT, Hormigo A, Yahalom J, et al. Long-term follow-up of high-dose methotrexate-based therapy 
with and without whole brain irradiation for newly diagnosed primary CNS lymphoma. J Clin Oncol. 2006;24:4570-4574.

[5] Poortmans PM, Kluin-Nelemans HC, Haaxma-Reiche $\mathrm{H}$, et al. High-dose methotrexate-based chemotherapy followed by consolidating radiotherapy in non-AIDSrelated primary central nervous system lymphoma: European organization for research and treatment of cancer lymphoma group phase II trial 20962. J Clin Oncol. 2003;21:4483-4488.

[6] Ferreri AJ, Reni M, Foppoli M, et al. High-dose cytarabine plus high-dose methotrexate versus high-dose methotrexate alone in patients with primary CNS lymphoma: a randomised phase 2 trial. Lancet. 2009;374:1512-1520.

[7] Ferreri AJ, Cwynarski K, Pulczynski $E$, et al. Chemoimmunotherapy with methotrexate, cytarabine, thiotepa, and rituximab (MATRix regimen) in patients with primary CNS lymphoma: results of the first randomisation of the international extranodal lymphoma study group-32 (IELSG32) phase 2 trial. Lancet Haematol. 2016;3:e217-e227.

[8] Doorduijn JK, van Imhoff GW, van der Holt B, et al. Treatment of secondary central nervous system lymphoma with intrathecal rituximab, high-dose methotrexate, and R-DHAP followed by autologous stem cell transplantation: results of the HOVON 80 phase 2 study. Hematol Oncol. Forthcoming 2016.

[9] Cheah CY, Joske D, Cull G, et al. High-dose therapy and autologous stem cell transplantation may only be applicable to selected patients with secondary CNS diffuse large B-cell lymphoma. $\mathrm{Br} J$ Haematol. Forthcoming 2016.

[10] Soussain C, Hoang-Xuan K, Taillandier L, et al. Intensive chemotherapy followed by hematopoietic stem-cell rescue for refractory and recurrent primary CNS and intraocular lymphoma: societe francaise de greffe de moelle osseuse-therapie cellulaire. J Clin Oncol. 2008;26:2512-2518.

[11] Korfel A, Elter T, Thiel E, et al. Phase II study of central nervous system (CNS)-directed chemotherapy including high-dose chemotherapy with autologous stem cell transplantation for CNS relapse of aggressive lymphomas. Haematologica. 2013;98:364-370.

[12] Damaj G, Ivanoff S, Coso D, et al. Concomitant systemic and central nervous system non-Hodgkin lymphoma: the role of consolidation in terms of high dose therapy and autologous stem cell transplantation. A 60-case retrospective study from LYSA and the LOC network. Haematologica. 2015;100: 1199-1206.

[13] Ferreri AJ, Donadoni G, Cabras MG, et al. High doses of antimetabolites followed by high-dose sequential chemoimmunotherapy and autologous stem-cell transplantation in patients with systemic B-cell lymphoma and secondary CNS involvement: final results of a multicenter phase II trial. J Clin Oncol. 2015;33: 3903-3910.

[14] Yoo KH, Lee JY, Lim SH, et al. Pilot trial of systemic methotrexate plus $\mathrm{R}-\mathrm{CHOP}$ regimen with intrathecal methotrexate for simultaneous central nervous system and systemic diffuse large B cell lymphoma. Acta Haematol. 2015;133:179-182.

[15] Chihara D, Fowler $\mathrm{NH}$, Oki $\mathrm{Y}$, et al. Dose-adjusted EPOCH-R and mid-cycle high dose methotrexate for patients with systemic lymphoma and secondary CNS involvement. Br J Haematol. Forthcoming 2016.

[16] Cheson BD, Pfistner $B$, Juweid $M E$, et al. Revised response criteria for malignant lymphoma. J Clin Oncol. 2007;25:579-586.

[17] Abrey LE, Batchelor TT, Ferreri AJ, et al. Report of an international workshop to standardize baseline evaluation and response criteria for primary CNS lymphoma. J Clin Oncol. 2005;23:5034-5043.

[18] Maciocia P, Badat M, Cheesman S, et al. Treatment of diffuse large B-cell lymphoma with secondary central nervous system involvement: encouraging efficacy using CNS-penetrating R-IDARAM chemotherapy. Br J Haematol. 2016;172:545-553.

[19] Riemersma SA, Jordanova ES, Schop RF, et al. Extensive genetic alterations of the HLA region, including homozygous deletions of HLA class II genes in B-cell lymphomas arising in immune-privileged sites. Blood. 2000;96:3569-3577.

[20] Nakamura T, Tateishi K, Niwa T, et al. Recurrent mutations of CD79B and MYD88 are the hallmark of primary central nervous system lymphomas. Neuropathol Appl Neurobiol. 2016;42:279-290.

[21] Berghoff AS, Ricken G, Widhalm G, et al. PD1 (CD279) and PD-L1 (CD274, B7H1) expression in primary central nervous system lymphomas (PCNSL). Clin Neuropathol. 2014;33:42-49.

[22] Houillier C, Choquet S, Touitou V, et al. Lenalidomide monotherapy as salvage treatment for recurrent primary CNS lymphoma. Neurology. 2015;84:325-326.

[23] Chamberlain MC. Salvage therapy with bendamustine for methotrexate refractory recurrent primary CNS lymphoma: a retrospective case series. J Neurooncol. 2014;118:155-162.

[24] Dunleavy K, Catherine EL, Roschewski M, et al. Phase I study of dose-adjusted-teddi-R with ibrutinib in untreated and relapsed/refractory primary CNS lymphoma. Blood. 2015;126:472.

[25] Lesokhin AM, Ansell SM, Armand P, et al. Nivolumab in patients with relapsed or refractory hematologic malignancy: preliminary results of a phase ib study. J Clin Oncol. 2016;34:2698-2704.

[26] Berghoff AS, Venur VA, Preusser $M$, et al. Immune checkpoint inhibitors in brain metastases: from biology to treatment. Am Soc Clin Oncol Educ Book. 2016;35:e116-e122. 